\title{
Health Economic Implications of Remote Patient Management
}

\author{
Mitchell H. Rosner • Sana Khan \\ Division of Nephrology, University of Virginia Health System, Charlottesville, VA, USA
}

\begin{abstract}
Remote patient management and telemedicine have great potential to improve the care of patients with end-stage kidney disease undergoing peritoneal dialysis (PD). Since PD is performed by patients and their families, typically in the home setting away from expert clinical support, remote monitoring of treatments may detect early problems, ensure compliance, and give both providers and patients a sense of reassurance that complications can be minimized and detected. These advantages may translate into improved outcomes and increased uptake of home dialysis therapies. Estimates of the financial impact of these potential improvements in care can be performed and are discussed in this chapter. These economic impacts are potentially significant and further the case for carefully designed clinical trials to assess the impact of these new technologies.

(c) 2019 S. Karger AG, Basel
\end{abstract}

\section{Introduction}

The incidence and prevalence of the end-stage kidney disease (ESKD) population continues to increase annually. Global utilization of peritoneal dialysis (PD) as a modality of renal replacement therapy varies widely, with most countries reporting PD utilization in $<22 \%$ of their dialysis patients [1]. The clinical outcomes of PD appear to be similar to those of hemodialysis [2-4]. PD continues to be less costly on a per patient basis compared to hemodialysis (USD 76,177 vs. 90,971 annually) [1]. Despite a gradual uptrend noted since 2011, 
utilization of PD across the United States appears to be low [1,5-7]. Several regulations have been observed to aid in increased $\mathrm{PD}$ utilization, including dialysis reimbursement and coverage of telehealth services [8,9]. In January 2016, legislation was passed mandating coverage of telemedicine for PD patients residing outside of a metropolitan area, or in a healthcare provider shortage area [9]. More recently, an Act passed in 2018 has eliminated the geographical restriction for telehealth provision, in an effort to improve home dialysis utilization [10]. There are many potential advantages to the utilization of telehealth for the management of PD patients. This chapter covers an overview of remote patient management (RPM) and telehealth, outcome data of telehealth programs, as well as subsequent economic advantages to global healthcare systems.

\section{Overview of RPM and Telehealth}

The term "RPM" is used to describe digital technologies that collect medical and other forms of health data from individuals in one location and electronically transmit that information securely to healthcare providers in a different location for assessment and recommendations [11]. "Telehealth" is a broader term that refers to the entire industry, methodology, and technologies that enable that type of healthcare $[12,13]$. RPM, then, is a type of telehealth delivery system. And the term telehealth may, in turn, refer to RPM - but it may also be used to refer to a great deal of other aspects of remote care.

The care pathway involved in the use of RPM or the broader technique of telehealth is relatively simple. Embedded in the home care environment are a series of technologies that might include: real-time video, 2-way communication

and a set of electronic sensors and devices that allow capture of diverse biometric data (including typical vital signs [blood pressure, pulse, respiratory rate, and temperature] as well as data on blood glucose, pulse oximetry, activity levels, gait stability, and other variables) [8]. In the realm of $\mathrm{PD}$, data that may be captured can also include various treatment parameters such as types of dialysis solutions utilized, duration of therapy, ultrafiltration amounts, drain times, and alarm types [8]. This information can be captured continuously, stored for later analysis, or sent on "demand" when requested by care providers. Data are typically sent to a central station where care providers view and analyze information and then make decisions such as prescription changes, education and other interventions. The ultimate purpose is providing remote support for the patient and their caregivers in the home or other environment with the goal of improving outcomes and decreasing cost of care [14]. 
As applied to PD, an RPM/telehealth system could be used to troubleshoot problems such as poor catheter flow, allow for monthly assessments by the care team in the home environment thus removing the burden of travel to a central clinic location, allow for treatment monitoring and assessment of compliance, provide laboratory data assessment and allow for continued patient education and direct visualization of patient technique $[8,14,15]$. Thus, the mission of an $\mathrm{RPM} /$ telehealth program, as applied to $\mathrm{PD}$, would be to allow for the realization of the promise of home therapies to increase independence, improve the quality of life, and increase acceptance of these modalities of renal replacement therapy.

Several systems have been designed and implemented that achieve various aspects of this mission; for example: [1] a tablet-based computer system that allows for patient-entered treatment data transmission as well as real-time visual chat between patients and clinicians and [2] a PD-cycler embedded RPM platform that allows healthcare providers to securely view their patients' recently completed home dialysis-related treatment data $[16,17]$. This last system is now commercially widely available (SHARESOURCE ${ }^{\mathrm{TM}}$; Baxter Healthcare, Chicago, IL, USA) and allows for all treatment data to be collected automatically after each PD session [17]. Healthcare providers can then act on this information by remotely adjusting their patients' home device settings, and the goal is to allow for early diagnosis and intervention of PD-related issues.

\section{Outcome Data for RPM/Telehealth Programs}

Given that RPM/telehealth programs for home dialysis are relatively new, outcome studies are limited and are, in general, small single center studies focused more on patient satisfaction and technique feasibility $[8,14]$. In terms of data that can link outcomes with a clear economic impact, there are a few notable studies in the literature. For example, Gallar et al. [18] evaluated the use of telemedicine in the long-term control of stable patients undergoing PD at home. Patients were randomly selected from current cases and invited to join study group A, in which they had telemedicine support. Patients not selected for this group, or who refused the invitation, were placed in study group B, and used for comparison. There were 25 patients in group A and 32 patients in group B. Videoconferencing equipment was installed in each patient's home and connected to a videoconferencing unit at the hospital. The mean hospitalization rate was 2.2 days/patient/year in group A and 5.7 days/patient/year in group B $(p<0.05)$. Dey et al. [19] provided PD patients with computer tablets that contained a knowledge database with treatment- and symptom-based questionnaires that generated alerts for the clinical team. Alerts were reviewed daily and followed 
up by a telephone call or clinic visit. Interventions were at the discretion of clinicians. Overall, 22 patients have participated over 15 months. A total of 1,195 alerts were received with an average of 2.6 alerts per day. A total of 36 admissions were avoided, and patients supported to self-manage on 154 occasions. More recently, an Italian group utilized the SHARESOURCETM ${ }^{\mathrm{TM}}$ platform in 37 patients undergoing PD [16]. They observed that patients utilizing this resource required less clinic visits and had their prescriptions modified more frequently. Data on hospitalizations and other healthcare utilization were not provided in this study.

In addition, there are data from other disease states where telehealth programs have been operating for extensive periods of time in larger populations [20]. A review of this data is beyond the scope of this article but an example of such data is a study from the United States Veterans Health Administration [20]. They assessed the efficacy of care coordination home telehealth to reduce the need for institutional care. After 12 months of enrollment in the telehealth program, there was a reduction of $4 \%$ in the mean annual healthcare costs, as well improved medication compliance. Several healthcare cost drivers (e.g., ER visits and admissions) had sizable declines in the telehealth group. Whether these dramatic effects can occur in other patient populations is not known but certainly the magnitude of these benefits is potentially of great significance.

\section{Can RPM/Telehealth Increase the Uptake of Peritoneal Dialysis?}

As of 2016, the percentage of ESKD utilizing PD as their renal replacement modality varied dramatically across the world. For most Western developed nations (United States and European Union), this percentage was approximately 10$15 \%[1,21]$. Countries in the Far East tend to have higher PD utilization rates $(20-40 \%)[1,21]$. Thus, there are clear barriers to the wider acceptance and use of PD in certain countries.

How might RPM and telehealth address the concerns and barriers associated with wider use of PD? As detailed by Oliver et al. [22], the barriers to PD use include: lack of acceptance and education among physicians, social barriers such as poor housing, lack of family and friend support, cognitive barriers, medical contraindications, and difficulty in arranging travel to the needed periodic assessments by the care team. RPM/telehealth has the ability to provide remote support for patients that addresses many of these barriers and concerns $[23,24]$. With RPM/telehealth, patients maintain autonomy but also have enhanced levels of support, supervision, and oversight. This may give both patients and providers a greater sense of relief that there is a ready support network that can step in at any time to help patients. Thus, RPM/telehealth provides the potential to 
Table 1. The economic impact of increasing PD uptake in the European Union

\begin{tabular}{|c|c|c|c|c|c|c|}
\hline Scenario & HD patients & PD patients & $\begin{array}{l}\text { Cost HD, } \\
\text { million } \\
\text { EUR }\end{array}$ & $\begin{array}{l}\text { Cost PD, } \\
\text { million } \\
\text { EUR }\end{array}$ & $\begin{array}{l}\text { Total cost, } \\
\text { million } \\
\text { EUR }\end{array}$ & $\begin{array}{l}\text { Cost savings, } \\
\text { million } \\
\text { EUR }\end{array}$ \\
\hline $\begin{array}{l}\text { PD: } 10 \% \\
\text { IHD: } 90 \%\end{array}$ & 328,500 & 36,500 & 101,923 & 6,013 & 107,936 & \\
\hline $\begin{array}{l}\text { PD: } 20 \% \\
\text { IHD: } 80 \%\end{array}$ & 292,000 & 73,000 & 90,599 & 12,026 & 102,625 & 5,311 \\
\hline $\begin{array}{l}\text { PD: } 30 \% \\
\text { IHD: } 70 \%\end{array}$ & 255,500 & 109,500 & 79,274 & 18,039 & 97,313 & 10,623 \\
\hline \multicolumn{7}{|c|}{$\begin{array}{l}\text { Analysis includes cost of telemedicine with an assumption of a } 5 \text {-year equipment lifetime } \\
\text { and a discount rate of } 4 \% / \text { year with approximate costs of EUR } 3,000 \text { per patient per year } \\
\text { (2017 estimates) [18]. } \\
\text { PD, peritoneal dialysis. }\end{array}$} \\
\hline
\end{tabular}

Table 2. The economic impact of increasing PD uptake in the United States [19]

\begin{tabular}{ll}
\hline Scenario & Cost savings to Medicare, million USD \\
\hline PD: $11.7 \%$ & \\
HHD: $3.3 \%$ & 114.8 \\
IHD: $85 \%$ & \\
\hline PD: $15.7 \%$ & \\
HHD $4.3 \%$ & 232.9 \\
IHD: $80 \%$ & \\
PD: $19.7 \%$ & \\
HHD: $5.3 \%$ & 350.9 \\
IHD: $75 \%$ & \\
\hline
\end{tabular}

address concerns that the patient may need additional oversight to ensure safety. Indeed, in a recent study in 300 participants in an RPM program, there was an improvement in perceived autonomy and confidence in healthcare activities and decreased negative perceptions of PD care and ESKD [25]. This may be enhanced by allowing real-time 2 -way conversations with video between patients and caregivers where problems and solutions are addressed as they occur and alleviate the wait time usually encountered to solve issues [14]. In addition, telehealth platforms allow for continuous education of patients and their caregivers with the ability to observe PD exchanges and technique. In all, RPM/telehealth provides for an increased level of oversight and education that could make a difference in allowing both providers and patients to be more comfortable with PD. 
In addition, the ability to provide care in the home through RPM/telehealth alleviates the need and associated costs of patient travel.

Economically, a shift towards higher utilization of PD yields large healthcare savings (Tables 1,2 ) as the provision of PD is far less expensive than hemodialysis [26, 27]. This is due to savings on labor and supplies. Thus, if RPM/telehealth allows for greater utilization of $\mathrm{PD}$, there are clear financial savings that accrue to healthcare systems even after paying for the infrastructure to support the provision of these modalities of care.

\section{Can RPM/Telehealth Lead to Better Outcomes and Lower Costs for PD Patients?}

RPM/telehealth have the ability to improve outcomes in ESKD patients. For instance, one could envision that RPM/telehealth could reduce the number of preventable admissions related to the dialysis modality such as those due to peritonitis, catheter exit site, volume overload and related to dialysis catheter malfunction [28]. Certainly, RPM/telehealth allows monitoring of patients to detect problems that can potentially be treated in the home environment. As an example, vital signs and daily weights can be sent to the dialysis center for review by the care team [29]. If the team notices an increase in weight, change in oxygen saturation levels or other symptoms, the patient can be contacted immediately and changes can be made in their PD prescription (such as using higher dextrose percentage bags) to increase the ultrafiltration amount and thus alleviate the risk for significant, symptomatic volume overload that might necessitate a trip to the hospital. As another example, a patient would have the ability to send real-time photos or videos of their catheter exit site to the care team if they noticed any redness or discharge that could be an early sign of infection. In this way, an exit site infection can be diagnosed early and treated in the home environment.

It is important to note that over $70 \%$ of hospital admissions in patients on $\mathrm{PD}$ are not directly related to the dialysis therapy itself and represent the effects of the numerous comorbidities that these patients suffer from such as diabetes mellitus, cardiovascular disease, and cancer [30]. Thus, RPM/telehealth programs for these patients should also address the broader care issues in these patients. This would include items such as continuous glucose monitoring with adjustments in medications by the care team that could be done remotely and more timely [31]. The RPM/telehealth platform could be configured in multiple ways to address a myriad of health problems with the promise that problems could be identified earlier as well as treated effectively in the home environment. 


\section{Reduction in Hospital Readmission Rates with RPM and Telehealth}

Readmissions are potentially modifiable and incur excess costs to health systems. Recent data from Canada revealed that the readmission rate for patients undergoing PD was 7.1 per 1,000 patient-days [32]. Interestingly, the readmission rates for PD patients were 19\% higher than hemodialysis patients. The reason hypothesized for this difference in readmission rates between dialysis modalities is that PD patients have fewer health system encounters and thus do not benefit from early interventions. This is in distinction to hemodialysis patients who are seen thrice weekly and thus have numerous opportunities for interventions to prevent readmissions. Thus, this is a clear area where RPM/ telehealth may prove beneficial. Patients can be followed with RPM to assess stability in vital signs, weights, and glycemic control [14]. Medications postdischarge can be reconciled in the home environment through 2-way communications [33]. Wound healing can be visually followed with the use of cameras. There is ample data from telehealth programs for conditions such as heart failure that have demonstrated significant reductions in readmission rates [34]. While not directly studied for patients on PD, there is no reason to suspect that similar results would not be seen in this group. However, this will require further study.

Quantifying the cost savings associated with reduction of readmissions is difficult but certainly this is a goal that improves the overall care. In countries, such as the United States, where reimbursement penalties exist for excessive readmission rates, there is likely some significant cost savings associated with these programs [35].

\section{Case Example}

A 23-year-old female with recent onset of ESKD starts PD with a cycler at home [36]. RPM is embedded in the cycler (CLARIA-SHARESOURCETM, Baxter Healthcare, Chicago, IL, USA) and is used to monitor the treatment. Her Initial course is unremarkable but within a few weeks, monitoring system "red flags" prolonged drain times. The clinical staff troubleshoots the problem and excludes issues such as constipation. Examination of the PD catheter reveals that malposition and surgical repositioning occurs with resolution of the problem. RPM continues and reveals that the patient misses her treatment at least 4-6 times per month. This leads to a discussion between the patient and her care team regarding compliance issues and education on the need to meet clearance goals. Compliance subsequently improves. 
This case illustrates the power of RPM and ongoing real-time interactions with a care team in improving outcomes. The early detection of a catheter malfunction likely led to a timely resolution of the issue that may have not occurred without RPM. Without RPM, there would have likely been a delay in diagnosis of the problem and a greater risk of severe complications (electrolyte problems, fluid overload, poor clearance, and others). In addition, RPM allows for detection of non-compliance which is clearly associated with poorer outcomes [37]. Non-compliant patients can be rapidly identified and counseled. Overall, RPM allowed for improved outcomes and lower costs in this case example.

\section{Summary}

RPM and telehealth offer patients and their care teams a set of tools that can enhance outcomes and lower costs. For the PD patient, these tools allow for the realization of the promise of home therapies to increase independence and improve outcomes. It is important to realize that these technologies must be embedded in care pathways and procedures that use these tools as enablers to maximize care. They are most effective when the care team knows how to use the data and procedures are developed to intervene effectively and rapidly. Data on outcomes associated with the use of RPM and telehealth in PD patients are lacking, and large randomized trials are needed to further our knowledge base. In addition, these data are needed to convince insurance carriers and governmental payers of the need to financially support these programs. The promise of improved outcomes and lower costs can be realized with these tools. In a recent simulation study, 12 automated PD profiles were examined for potential savings in healthcare resource use [38]. The estimated reduced utilization across the 3 countries ranged from 1 to 2 hospitalizations, $1-4$ home visits, $2-5$ emergency room visits, and $4-8$ unplanned clinic visits. Total savings across all scenarios were USD 23,364 in the United States, USD 11,477 in Germany, and USD 7,088 in Italy. While this is a simulation study, it highlights the potential benefit that broader utilization of RPM/telehealth resources may provide. 


\section{References}

1 United States Renal Data System 2018. https:// www.usrds.org/adr.aspx (accessed November 30, 2018).

2 Khawar O, Kalantar-Zadeh K, Lo WK, et al: Is the declining use of long-term peritoneal dialysis justified by outcome data? Clin J Am Soc Nephrol 2007;2:1317-1328.

3 Chiu YW, Jiwakanon S, Lukowsky L, et al: An update on the comparisons of mortality outcomes of hemodialysis and peritoneal dialysis patients. Semin Nephrol 2011;31:152-158.

4 Mehrotra R, Chiu YW, Kalantar-Zadeh K, et al: Similar outcomes with hemodialysis and peritoneal dialysis in patients with end-stage renal disease. Arch Intern Med 2011;171:110-118.

5 Rivara MB, Mehrotra R: The changing landscape of home dialysis in the United States. Curr Opin Nephrol Hypertens 2012;178:79-82.

6 Morton RL, Snelling P, Webster AC, et al: Dialysis modality preference of patients with CKD and family caregivers: a discrete-choice study. Am J Kidney Dis 2012;60:102-111.

7 Wang V, Coffman CJ, Sanders LL, et al: Medicare's new prospective payment system on facility provision of peritoneal dialysis. Clin J Am Soc Nephrol 2018;13:1833-1841.

8 Rosner MH, Lew SQ, Conway P, et al: Perspectives from the kidney health initiative on advancing technologies to facilitate remote monitoring of patient self-care in RRT. Clin J Am Soc Nephrol 2017;12:1900-1909.

9 Centers for Medicare and Medicaid Services. TeleHealth Services. https://www.cms.gov/Outreach-and-Education/Medicare-Learning-Network-MLN/MLNProducts/downloads/TelehealthSrvcsfctsht.pdf (accessed December 5, 2018).

10 S.870-Creatining-High-Quality Results and Outcomes Necessary to Improve Chronic (CHRONIC) Care Act of 2017. https://www.congress.gov/ bill/115th-congress/senate-bill/870.

11 Coye M, Haskelkorn A, Demello S: Remote patient management: technology-enabled innovation and evolving business models for chronic disease care. Health Aff (Millwood) 2009;28:126135.

12 https://www.mayoclinic.org/healthy-lifestyle/ consumer-health/in-depth/telehealth/art20044878 (accessed November 30, 2018).

13 American Telemedicine Association: What is Telemedicine? http://www.americantelemed.org/ about-telemedicine/what-is-telemedicine\#. V1RDcpMrKu4. (accessed November 30, 2018).

14 Krishna VN, Managadi K, Smith M, Wallace E: Telehealth in the delivery of home dialysis care: catching up with technology. Adv Chronic Kidney Dis 2017;24:12-16.
15 Harrington DM, Myers L, Eisenman K, et al: The use of a tablet computer platform to optimize the care of patients receiving peritoneal dialysis: a pilot study. Blood Purif 2014;37:311-315.

16 Milan Manani S, Crepaldi C, Giuliani A, et al: Remote monitoring of automated peritoneal dialysis improves personalization of dialytic prescription and patient's independence. Blood Purif 2018;46:111-117.

17 https://www.baxter.com/healthcare-professionals/renal-care/amia-sharesource-renal-care (accessed November 30, 2018).

18 Gallar P, Vigil A, Rodriguez I, et al: Two-year experience with telemedicine in the follow-up of patients in home peritoneal dialysis. J Telemed Telecare 2007;13:288-292.

19 Dey V, Jones A, Spalding EM: Telehealth: acceptability, clinical interventions and quality of life in peritoneal dialysis. SAGE Open Med 2016;4:1-6.

20 Darkins A: Telehealth Services in the United States Department of Veterans Affairs. 2014 https://www.graham-center.org/content/dam/ rgc/documents/press-events/primary-care-forum/AdamDarkins-Telehealth.pdf (accessed November 30, 2018).

21 Jain AK, Blake P, Cordy P, Garg AX: Global trends in rates of peritoneal dialysis. J Am Soc Nephrol 2012;23:533-544.

22 Oliver MJ, Garg AX, Blake PG, et al: Impact of contraindications, barriers to self-care and support on incident peritoneal dialysis utilization. Nephrol Dial Transplant 2010;25:2737-2744.

23 Lew SQ: Telehealth in peritoneal dialysis: review of patient management. Adv Perit Dial 2018;34: 32-37.

24 Bieber SD, Weiner DE: Telehealth and home dialysis: a new option for patients in the United States. Clin J Am Soc Nephrol 2018:13:12881290.

25 Magnus M, Sikka N, Cherian T, Lew SQ: Satisfaction and improvements in peritoneal dialysis outcomes associated with telehealth. Appl Clin Inform 2017;8:214-225.

26 Pike E, Hamidi V, Ringerike T, et al: More use of peritoneal dialysis gives significant savings: a systematic review and health economic decision model. J Clin Med Res 2017;9:104-116.

27 Liu FX, Walton SM, Leipold R, et al: Financial implications to medicare from changing the dialysis modality mix under the bundled prospective payment system. Perit Dial Int 2014;34:749757.

28 Nayak KS, Ronco C, Karopadi AN, Rosner MH: Telemedicine and remote monitoring: supporting the patient on peritoneal dialysis. Perit Dial Int 2016;36:362-363. 
29 Lew SQ, Sikka N, Thompson C, Cherian T, Magnus M: Adoption of telehealth: remote biometric monitoring among peritoneal dialysis patients in the United States. Perit Dial Int 2017:37:576-578

30 Quinn RR, Ravani P, Zhang X, et al: Impact of modality choice on rates of hospitalization in patients eligible for both peritoneal dialysis and hemodialysis. Perit Dial Int 2014;34:41-48.

31 Lan T, Zhang J, Lu Y: Transforming the blood glucose meter into a general healthcare meter for in vitro diagnostics in mobile health. Biotechnol Adv 2016;34:331-341.

32 Perl J, McArthur E, Bell C, et al: Dialysis modality and readmission following hospital discharge: a population-based cohort study. Am J Kidney Dis 2017;70:11-20.

33 Omboni S, Caserini M: Effectiveness of pharmacist's intervention in the management of cardiovascular diseases. Open Heart 2018;5:e000687.
34 Koehler F, Koehler K, Deckwart O, et al: Efficacy of telemedical interventional management in patients with heart failure (TIM-HF2): a randomised, controlled, parallel-group, unmasked trial. Lancet 2018;392:1047-1057.

35 Kripalani S, Theobald CN, Anctil B, Vasilevskis EE: Reducing hospital readmission rates: current strategies and future directions. Annu Rev Med 2014;65:471-485.

36 Drepper VJ, Martin PY, Chopard CS, Sloand JA: Remote patient management in automated peritoneal dialysis: a promising new tool. Perit Dial Int 2018;38:76-78.

37 Bernardini J, Nagy M, Piraino B: Pattern of noncompliance with dialysis exchanges in peritoneal dialysis patients. Am J Kidney Dis 2000;35:11041110.

38 Makhija D, Alscher MD, Becker S, et al: Remote monitoring of automated peritoneal dialysis patients: assessing clinical and economic value. Telemed J E Health 2018;24:315-323. 